\title{
Utilisation de myoblastes à des fins de thérapie génique
}

Nous avons déjà signalé dans ces colonnes [1] que les cellules satellites du muscle, qui peuvent être cultivées ex vivo comme des myoblastes, pouvaient servir de véhicules à des transgènes thérapeutiques. Après transfert de gènes, les myoblastes réinjectés dans le muscle fusionnent, en effet, entre eux et avec les myotubes pré-existant dans lesquels ils transfèrent ainsi leur noyau et leur transgène. Ce que l'on ne savait pas, en revanche, c'est si les cellules musculaires auraient la capacité de sécréter dans le sang une quantité significative d'une protéine synthétisée sous le contrôle du transgène. C'est une réponse positive à cette question qu'ont pu apporter deux équipes américaines dirigées, l'une par G.M. Leiden (Ann Harbor, MI) et l'autre par HM Blau (Stanford, CA) [2, 3]. La première équipe utilisa, pour ce faire, un plasmide alors que la seconde faisait appel à un rétrovirus, tous deux recombinés par intégration du gène de l'hormone de croissance. Dans les deux cas, ce furent les myoblastes de la lignée myogénique établie $\mathrm{C} 2 \mathrm{C} 12$ qui furent utilisés. Le destin des cellules réinjectées fut précisé par l'utilisation de cellules portant un transgène différent, celui codant pour la $\beta$ galactosidase dont l'activité est aisément repérable grâce à une réaction histochimique [1]. Certains myoblastes semblent éliminés, d'autres prolifèrent ; d'autres, enfin, fusionnent avec des myotubes pré-existants. Dans les deux cas, la sécrétion d'hormone de croissance dans le sang est aisément détectable, trois semaines [2] et trois mois [3] après l'injection. Ces résultats semblent donc très prometteurs, même si d'importantes difficultés restent probablement à surmonter. Tout d'abord, évidemment, il ne serait pas question, chez l'homme, d'utiliser des lignées établies, potentiellement tumorigènes, de myoblastes. Il faudra donc s'assurer que les myoblastes issus des cellules satellites des malades, ou encore une population non tumorigène et, idéalement, non immunogène de myoblastes en culture, donnent d'aussi bons résultats. Les données actuellement disponibles de la survie des myoblastes greffés chez des myopathes montrent que des améliorations sont encore nécessaires dans ce domaine [4]. Si les cellules greffées viennent d'un donneur non totalement compatible, se posera aussi le problème du traitement immunosuppresseur nécessaire à la persistance des cellules greffées. Lorsque toutes ces difficultés seront surmontées, car il semble peu douteux qu'elles soient surmontables, l'utilisation des myoblastes comme véhicules d'ADN thérapeutique aura probablement plus d'avenir lorsqu'il s'agira de sécréter dans le sang une hormone ou une protéine quelconque que lorsque le but sera de guérir une maladie musculaire congénitale telle que la myopathie. Dans ce dernier cas, en effet, il serait nécessaire de corriger une grande proportion des myotubes alors que la présence de quelques myotubes sécrétant la protéine thérapeutique peut être suffisante dans la première indication. Certains envisagent même la période où l'on pourra greffer à des diabétiques des myoblastes recombinés avec le gène de l'insuline placé sous le contrôle de régions régulatrices sensibles à la concentration du glucose sanguin [5].

A.K.

1. Kahn A, Briand P. Thérapie génique : espoirs et limites. médecine/sciences 1991; 7 : 705-14. 2. Parr E, Leiden JM. Systemic delivery of recombinant proteins by genetically modified myoblasts. Science 1991; 254 : 1507-9.
3. Dhawan J, Pan LC, Tavlath GK, Travis MA, Lancdot AM, Blau HM. Systemic delivery of human growth hormone by injection of genetically engineered myoblasts. Science $1991 ; 254$ : 1509-12.

4. Labrecque UEC, Bouchard JT, Malouin F, Roy R, Huard J, Tremblay JP. Approches thérapeutiques de la myopathie de Duchenne par transplantation de myoblastes. médecine/sciences 1991; 7 : 821-9.

5. Hoffman M. Putting new muscle into gene therapy. Science 1991; 254 : 1455-6.

\section{- COLLĖGE DE FRANCE • CHAIRE D'ANTHROPOLOGIE PHYSIQUE}

M. Jacques Ruffié, professeur Année 1991-1992

Séminaires avec la collaboration de Ph. Rouger

\section{L'individu biologique}

17 janvier : Bases génétiques de l'individu.

24 janvier : Le soi immunologique.

31 janvier: Les marqueurs immunogénétiques.

7 février: Le polymorphisme.

17 février : Le polymorphisme de I'ADN.

21 février : Les frontières de l'individu biologique.

28 février : Les analyses de filiation.

6 mars : Biologie et médecine légale.

13 mars : Approches actuelles de la définition des populations. Les séminaires auront lieu le vendredi, à 10 h 15, dans la salle 8 . Les cours portant sur "La Médecine prédictive" auront lieu aux mêmes dates et dans la même salle, de 9 heures à 10 heures.

L'Administrateur du Collège de France André Miquel 